\title{
Cuando $7+3=4$ parece correcto: resolución automática de las restas en una tarea de verificación
}

\author{
Valle Lara Carmona ${ }^{1}$, Javier García Orza ${ }^{1}$ y Patricia Carratalá Cepedal ${ }^{2}$ \\ ${ }^{1}$ Universidad de Málaga, ${ }^{2}$ I.E.S. Universidad Laboral de Málaga
}

Disponible online 30 de agosto de 2009

\begin{abstract}
Existen múltiples evidencias de que la resolución de multiplicaciones y sumas simples es un proceso automático para la mayoría de los adultos instruidos. Sin embargo, hay escasas pruebas de la automaticidad de otras operaciones como la resta o la división. En el presente estudio se analiza el grado de automaticidad de restas en las que ambos operandos son de una cifra. Diecisiete estudiantes de psicología tomaron parte en un experimento en el que debían juzgar si las sumas presentadas eran correctas o no. Entre las sumas incorrectas el resultado podía estar relacionado con los operandos mediante una relación de resta (e.g., 7+3=4) o no estar relacionado (e.g., 7+3=2). El análisis de los resultados muestra un efecto de interferencia, es decir, un peor rendimiento en la condición de resta comparada con la condición no relacionada. Los datos sugieren que la resolución de restas se caracteriza por un alto grado de automaticidad.
\end{abstract}

Palabras clave:

Resta, automático, verificación, interferencia, hechos aritméticos

There is a great amount of evidence showing that adults solve single-digit multiplications and additions automatically. Nevertheless, evidence regarding subtraction and division is scarce. The present study explores whether the solving of single-digit subtractions is automatic or not. Seventeen psychology students took part in our experiment. They have to judge the correctness of single-digit additions. In the "false additions" the result could be the subtraction of the operands (e.g., $7+3=4$ ) or could be unrelated with the operands (e.g., $7+3=2$ ). The data analysis showed the presence of an interference effect, this is, worse performance in the subtraction condition than in the unrelated condition. These results suggest that subtraction solving is highly automatic.

Keywords:

Rubtraction, automatic, verification, interference, arithmetical facts

\footnotetext{
El presente trabajo ha sido desarrollado en el marco del Proyecto PSI2008-06107/PSIC "Psicología y Neuropsicología Cognitiva del Procesamiento Numérico y el Cálculo" financiado por el Ministerio de Ciencia e Innovación.

Correspondencia: Javier Garcia Orza, Facultad de Psicología, Campus de Teatinos s/n, 29071, Málaga. Telf.: 952132982 , Fax: 952132631 jgorza@uma.es
} 
Las matemáticas están presentes en nuestra vida cotidiana y sin un mínimo dominio de ellas el ser humano se siente indefenso; sin ellas no se puede comprar, saber la hora, qué día es hoy, quién ganó un partido, o cuántos bombones quedan en la caja si tenía 8 y se han comido 2 . Afortunadamente, cualquier persona instruida es capaz de entender los números e incluso resolver sin dificultades los sencillos problemas matemáticos que acabamos de plantear. La psicología cognitiva lleva ya muchos años intentando explicar los procesos cognitivos implicados en el procesamiento numérico y en las diferentes operaciones aritméticas (ver Campbell, 2005, para una revisión reciente) y, sin embargo, el camino por recorrer es todavía largo pues nuestro conocimiento sobre tareas tan simples como decidir qué número de los que nos presentan es mayor o cuáles son los procesos implicados en la resolución de operaciones como la suma o la resta siguen siendo objeto de discusión.

Un área central en el estudio de la cognición matemática es la que aborda el estudio de los hechos aritméticos básicos (arithmetical facts), entendiendo por estos aquellas operaciones en las que ambos operandos y/o el resultado son de una cifra (p.ej., 3 x $5=15 ; 5+7=12 ; 17-8=9 ; 54 / 9=6$ ). Algunas investigaciones empleando autoinformes y/o el análisis de los tiempos de respuesta sugieren que los adultos emplean diversas estrategias para su resolución. Así, un problema como $2 \times 3$ se puede solucionar simplemente contando con los dedos o bien sumando $(2+2+2=6)$, es decir, aplicando un procedimiento, o mediante la recuperación directa de representaciones $(2 \times 3=6)$ que tenemos almacenadas en nuestra memoria (e.g., Campbell y Penner-Wilger, 2006). Este último mecanismo de resolución de las multiplicaciones parece ser la forma más común, al menos en adultos y en la mayoría de los niños (e.g., Hecht, 1999; LeFevre et al., 1996; Romero, Rickard y Bourne, 2006; Roussel, Fayol y Barrouillet, 2002), y parece tener lugar de forma automática (ver Galfano, Rusconi y Umiltà, 2003; y García-Orza, Damas-López, Matas y Rodríguez, 2009 para una revisión). Similares resultados se han encontrado con la suma (e.g., LeFevre, Bisanz y Mrkonjic, 1988; Lemaire, Barret, Fayol y Abdi, 1994) y, sin embargo, no está claro que la resolución de otros hechos aritméticos básicos como la división, y, sobre todo, la resta siga los mismos mecanismos. El objetivo del presente trabajo es estudiar si la resolución de las restas se produce también de forma automática, pues no existen hasta la fecha, que nosotros sepamos, evidencias sobre esta cuestión. De hecho, como señalaremos más adelante, suele asumirme que la resta, incluso de números de una cifra, implica procesos de conteo y cálculo y rara vez de recuperación automática (e.g., Dehaene y Cohen, 1995).

Las investigaciones que han intentado demostrar experimentalmente la automaticidad de los hechos aritméticos simples han empleado principalmente dos tipos de tareas, las tareas de verificación de operaciones y las tareas de emparejamiento numérico. Hace ya algunos años Winkelman y Schmidt (1974) y Zbrodoff y Logan (1986) mostraron que cuando se usa una tarea de verificación, en la que los sujetos deben decidir si las operaciones aritméticas que se les presentan son correctas o incorrectas, se tarda más en detectar errores del tipo 3+4=12 (ítem interferido por la representación $3 \times 4=12$ ) que errores como 3+4=18. Este fenómeno de interferencia conocido como "interferencia inter-operaciones" (cross-operation interference) sugiere que las multiplicaciones interfieren en el procesamiento de las sumas, es decir, que ante la visión de 3+4=12 se activaría de forma automática $3 \times 4=12$, que es correcto, lo que ralentiza el rechazo de la operación presentada en comparación con otra como $3+4=18$.

La activación automática de las multiplicaciones ha sido estudiada también por medio de la tarea de emparejamiento de números (number matching task), en la que se presentan dos números (e.g., 3 6) que son rápidamente sustituidos por un tercero (e.g., 18). Los sujetos deben decidir si el último número presentado es uno de los que se han mostrado inicialmente. Empleando este tipo de tareas, LeFevre et al. (1988) mostraron que cuando el último número presentado era la suma de los dos presentados inicialmente (e.g., 3 8...11) el rendimiento de los sujetos empeoraba en comparación con una condición en la que el último número no guardaba relación alguna con el par inicial (e.g., 38 ...12). Estudiando las multiplicaciones Thibodeau, LeFevre y Bisanz (1996) encontraron similares efectos: cuando el último número presentado era la solución de multiplicar el par de números inicial (e.g., 36 ... 18) los participantes en el experimento empleaban más tiempo en dar una respuesta negativa en comparación con una condición de control (e.g., 36 ... 25). Estos resultados se han interpretado como una evidencia de que la mera presentación de dos dígitos es capaz de activar el resultado de su suma o de su multiplicación, apoyando la automaticidad de estas operaciones.

Recientemente, García-Orza et al. (2009) han empleado una tarea diferente para investigar la automaticidad de las multiplicaciones. Presentaron a los sujetos una tarea de denominación de números arábigos los cuales iban precedidos de un prime enmascarado presentado muy brevemente y que podía ser una multiplicación congruente (e.g., $3 \times 4$ seguido de 12) o incongruente (e.g., 5x9 seguido de 12) con el número a denominar. A pesar de no ver las multiplicaciones conscientemente, los tiempos de denominación de los números fueron menores para los números que iban precedidos por una multiplicación congruente comparados con los precedidos por multiplicaciones incongruentes. Es decir, de alguna manera los sujetos ante la visión (inconsciente) de la operación son capaces de activar su resultado, la preactivación de ese resultado es aprovechada por el sistema de procesamiento numérico en las condiciones congruentes para facilitar la denominación del número presentado posteriormente.

Aunque las evidencias con la suma y la multiplicación descritas hasta ahora sugieren un alto grado de automaticidad, los estudios con las divisiones y las restas son escasos. Recientemente, De Brauwer y Fias (2007) usando la tarea de empareja- 
miento numérico han encontrado efectos de interferencia en la resolución de divisiones, lo que parece añadir esta operación al grupo de las que son resueltas por medio de la recuperación automática de información sobre las mismas en nuestra memoria. Por otro lado, la falta de estudios publicados sobre la recuperación automática de restas posiblemente se debe no a que no se hayan realizado, sino a que los hallazgos sean de ausencia de efectos de interferencia $y$, al describir por tanto un efecto nulo, no sean fácilmente publicables. Sin embargo, creemos que incluso en el caso de ser así, merece la pena conocer si efectivamente la resolución de estas operaciones es automática o no, y más como en el presente caso, cuando existe al menos un modelo de la cognición matemática que hace predicciones explícitas sobre esta cuestión. El Modelo de Triple-Código (Dehaene, 1992; Dehaene y Cohen, 1995), posiblemente el modelo de procesamiento numérico y de cálculo más importante en la actualidad, considera que las restas no se resuelven de forma automática habitualmente. En la resolución de las restas se hace uso de un código de magnitud de tipo analógico que es poco preciso, es decir, calcula las cantidades de forma más o menos aproximada, impidiendo por tanto la resolución de las restas de forma automática. Por el contrario, el modelo sí considera que la resolución automática de operaciones tiene lugar en la suma y la multiplicación y tendría lugar por la recuperación de secuencias verbales/tres por dos seis/memorizadas en nuestro aprendizaje escolar mediante asociación. El resto de los modelos (e.g., McCloskey, 1992; Cipolotti y Butterworth, 1995) simplemente considera que el grado de automatismo de las operaciones dependerá básicamente de su frecuencia de resolución, de forma que las restas más frecuentes se van aprendiendo por asociación, lo que facilitaría su recuperación de forma automática posteriormente. Es decir, para estos modelos no es difícil asumir el automatismo de las restas o la ausencia del mismo, pues este dependerá de la fuerza asociativa que la experiencia con tales operaciones genere.

Se describe a continuación un experimento que tiene como objetivo el estudio de la automaticidad de restas de una cifra a partir de una tarea de verificación. Usando el paradigma de interferencia entre operaciones, se presentan sumas correctas e incorrectas que el sujeto debe verificar. Entre las incorrectas el resultado puede ser el resultado de restar los dos operandos o bien un número no relacionado que actúa como control. Si la resolución de las restas es automática, entonces debe darse peor ejecución en la condición de relación de resta que en la de control, es decir, un efecto de interferencia entre operaciones. $\mathrm{Si}$, por el contrario, la resolución de restas no es automática no se esperan diferencias entre ambas condiciones.

Método

\section{Participantes}

Diecisiete estudiantes de psicología de la Universidad de Málaga participaron en el experimento de forma voluntaria. Sus edades estaban comprendidas entre los 19 y los 28 años, 14 eran mujeres y 3 varones. Todos los participantes desconocían el propósito del experimento.

\section{Materiales}

Se seleccionaron 10 operaciones de suma correctas del tipo $a+b$, donde "a" y "b" podían ser números entre 1 y 9 y "a" era siempre mayor que "b". En la condición de ítems correctos cada suma iba acompañada de su solución correcta, las 10 operaciones se presentaron dos veces. Para la condición de ítems incorrrectos se utilizaron las mismas 10 sumas, sólo que mientras que en una condición la solución podía estar relacionado con los sumandos mediante una relación de resta, es decir, la solución correspondía con la resta del primer y el segundo operando (e.g., $7+3=4$ ), en la otra condición, condición de control, no existía relación entre la solución y los operandos (e.g., $7+3=2$ ) (ver tabla 1). Con el objetivo de evitar diferencias entre ambas condiciones debido al efecto de distancia (en las tareas de verificación se rechazan tanto más rápidamente los ítems cuanto mayor sea la distancia entre la solución real y la presentada, e.g., Winkelman y Schmidt, 1974), las soluciones a los estímulos de control se eligieron de forma que la distancia entre la solución presentada y la solución real fuera la misma en términos medios entre las condiciones de relación de resta y de control. Junto a los ítems descritos se incluyeron 10 ítems de relleno tanto en la condición de ítems correctos como en la de ítems incorrectos, los cuales además se presentaron dos veces. En resumen, el experimento estaba constituido por 20 sumas correctas ( 10 experimentales y 10 de relleno) que se presentaban dos veces y 30 incorrectas (10 relacionadas por resta, 10 de control y 10 de relleno, presentándose estas últimas 2 veces). De esta forma los participantes veían 40 operaciones correctas y 40 incorrectas. Seis operaciones, tres correctas y tres incorrectas, construidas para la ocasión se utilizaron como ítems de prácticas.

Tabla 1. Sumas correctas e incorrectas para la tarea de verificación. En las incorrectas se presentan las de relación de resta y la condición de control las cuales constituyen las condiciones críticas para el experimento. No se incluyen los materiales de relleno.

\begin{tabular}{ccc}
\hline Respuesta Correcta & \multicolumn{2}{c}{ Respuesta Incorrecta } \\
\hline & Relación de resta & Condición de Control \\
\hline $7+3=10$ & $7+3=4$ & $7+3=5$ \\
$9+2=11$ & $9+2=7$ & $9+2=6$ \\
$8+1=9$ & $8+1=7$ & $8+1=6$ \\
$9+4=13$ & $9+4=5$ & $9+4=6$ \\
$5+1=6$ & $5+1=4$ & $5+1=3$ \\
$6+2=8$ & $6+2=4$ & $6+2=5$ \\
$8+6=14$ & $8+6=2$ & $8+6=1$ \\
$7+4=11$ & $7+4=3$ & $7+4=2$ \\
$6+4=10$ & $6+4=2$ & $6+4=3$ \\
$5+2=7$ & $5+2=3$ & $5+2=4$ \\
\hline
\end{tabular}




\section{Procedimiento}

Los participantes se sentaron frente a un monitor de 15 " y a un ordenador equipado con ERTS (Beringer, 1999) el cual controlaba la presentación de los estímulos y registraba los tiempos de respuesta a un nivel de precisión de milisegundo. Inicialmente se presentaban tres pantallas en las que se proporcionaban las instrucciones a los participantes. Éstas señalaban que se iban a presentar sumas junto a sus soluciones y ellos debían juzgar apretando las teclas designadas para ello si tales operaciones eran correctas o no. Las instrucciones enfatizaban que se debía responder lo más rápidamente posible y evitar los errores. Cada ensayo comenzaba con la presentación de un punto de fijación durante $500 \mathrm{~ms}$, inmediatamente después aparecía una suma seguida del signo igual y la solución propuesta, las cuales permanecían en la pantalla hasta la emisión de la respuesta o un máximo de $5000 \mathrm{~ms}$. Los números se presentaron en formato NRC 7bits tamaño de 16 puntos. El experimento se desarrollaba en una sola sesión cuya duración era aproximadamente de 10 minutos.

\section{Resultados}

Los análisis se realizaron exclusivamente sobre las respuestas de los sujetos a los items en los que el resultado de la operación era incorrecto, pues son las que permiten analizar la automaticidad del procesamiento de las restas. En la tabla 2 se ofrecen las medias de los tiempos de respuesta y de los errores en las condiciones experimentales de interés: relación de resta y relación de control.

Las respuestas erróneas (6'1\%) y aquellos tiempos mayores de $3000 \mathrm{~ms}$ (menos del 5\% de los datos) fueron excluidos del análisis de tiempos de respuesta. Se realizó una prueba de comparación de medias entre la condición de resta (e.g., $7+3=4$ ) y de control (e.g., $7+3=2$ ). Los resultados muestran que la diferencia de $64 \mathrm{~ms}$ favorable a la condición de control resultó significativa, $\mathrm{t}(16)=2.43 ; \mathrm{p}=.027$; I.C.95\%: $8.04,119.5$; $\eta^{2}=0.27$

Tabla 2. Media de los tiempos de respuesta (en ms.) y del porcentaje de errores en las condiciones de relación de resta y control (entre paréntesis las desviaciones típicas).

\begin{tabular}{ccc}
\hline Condición & Tiempos de Respuesta & Porcentaje de errores \\
\hline Relación de Resta & $1225(221)$ & $3.8(5.9)$ \\
Control & $1161(236)$ & $2.3(3.1)$ \\
\hline
\end{tabular}

El análisis de los errores muestra que aunque la proporción de errores en la condición de relación de resta era mayor que en la condición de control, siguiendo la tendencia encontrada en el análisis de los tiempos de respuesta, esta vez las diferencias no fueron significativas, $\mathrm{t}(16)=0.92 ; \mathrm{p}=.37$; I.C.95\%:-0.02, 0.05 ; $\eta^{2}=0.05$.

\section{Discusión General y Conclusiones}

Es asumido por muchos investigadores (e.g., Dehaene y Cohen, 1995), pero también por muchas personas (e.g., Romero et al., 2006), que las restas de una cifra suelen implicar procesos de conteo y no se resuelven de forma automática. En el presente estudio hemos analizado el grado de automatismo de las restas mediante una tarea de verificación empleando un paradigma de interferencia entre operaciones. Los resultados no dejan lugar a dudas: ante respuestas incorrectas en las que el resultado es la resta de los operandos los sujetos tardan más en calificar como incorrecta esa operación que ante una no relacionada. Este hallazgo de interferencia entre operaciones sugiere que los sujetos son capaces de activar que, con otro signo (un menos en vez de un más), tal respuesta sería correcta y por tanto tardan más en rechazarla. Las restas son calculadas de forma rápida y sin intención a pesar de perjudicar el rendimiento de los sujetos en la tarea, es decir, la resolución de las restas presenta las propiedades que definen los procesos automáticos, rapidez e involuntariedad (ver Zbrodoff y Logan, 1986). Al menos en adultos, parece existir una asociación directa entre los dígitos de una cifra y sus restas que es activada inmediatamente, sin intención y que produce, en el marco de una tarea de verificación, un claro efecto de interferencia.

Nuestros resultados parecen mostrar que las restas deben añadirse al resto de las operaciones aritméticas básicas, que se caracterizan por el hecho de que cuando están formadas por operandos con una cifra, la solución a tales operaciones de suma, multiplicación y división se activa de forma automática.

Aunque el hallazgo de efectos de interferencia es contrario a las predicciones del modelo de triple código (Dehaene, 1992; Dehaene y Cohen, 1995), el modelo podría asumir estos resultados de forma sencilla: basta con considerar que el repetido encuentro con las restas va generando por asociación unas representaciones verbales de la relación entre operandos y su solución (/cinco menos tres son dos/), que son posteriormente recuperadas de forma automática de nuestra memoria. Es decir, ya no habría necesidad de utilizar en el caso de la resta un código de magnitud de tipo analógico mediante el cual realizar el proceso de estimación del resultado, tal y como se defiende en el modelo original.

No queremos finalizar sin apuntar dos cuestiones relacionadas con el alcance de los hallazgos de la presente investigación. La conclusión de que la solución a las restas se recupera de forma automática puede generalizarse a la población objeto de este estudio, estudiantes universitarios. Sin embargo, queda por determinar hasta qué punto es automático el procesamiento de las restas en personas sin estudios universitarios o, por ejemplo, en niños en los que el aprendizaje de estas operaciones sea muy reciente. Por otra parte, las restas que se han utilizado en el presente estudio son las más simples posibles, los operandos eran siempre de una sola cifra 
(e.g., 7-3=4). Queda por determinar si restas simples pero en las que uno de los dos operandos sea de dos cifras (e.g., 14-6=8) serían resueltas también de forma automática. En relación con estas dos cuestiones, existen evidencias en la literatura que muestran la importancia de las diferencias individuales en el cálculo, y otras de tipo evolutivo que sugieren que lógicamente el proceso de automatización, al menos en sumas y multiplicaciones, es progresivo en niños (Lemaire et al., 1994). También hay datos que sugieren que la magnitud de los números implicados en las operaciones es una variable importante. Así LeFevre et al. (1988) encontraron mayor efecto de interferencia cuanto menores eran los operandos, es decir, la recuperación de la solución es más automática cuanto menores son los operandos. Investigaciones futuras nos permitirán estudiar hasta qué punto son aplicables los hallazgos citados, realizados con la suma y la multiplicación, al caso de la resta.

\section{Referencias}

Beringer, J. (1999). Experimental run time system (ERTS). Version 3.28. Frankfurt: Berisoft.

Campbell, J. I. D. (2005). Handbook of mathematical cognition. New York: Psychology Press.

Campbell, J. I. D. y Penner-Wilger, M. (2006). Calculation latency: The $\mu$ of memory and the $\tau$ of transformation. Memory \& Cognition, 34, 217-226.

Cipolotti, L. y Butterworth, B. (1995). Towards a multiroutte model of number processing: Impaired number transcoding with preserved calculation skills. Journal of Experimental Psychology: General, 124, 375-390.

Dehaene, S. (1992). Varieties of numerical abilities. Cognition, $44,1-42$.

Dehaene, S. y Cohen, L. (1995). Towards an anatomical and functional model of number processing. Mathematical Cognition, 1(1), 83-120.

De Brauwer, J. y Fias, W. (2007). The memory representation of multiplication and division facts. Comunicación presentada al XV Meeting de la European Society for Cognitive Psychology. Agosto, Marsella, Francia.

Galfano, G., Rusconi, E. y Umiltà, C. (2003). Automatic activation of multiplication facts: evidence from the nodes adjacent to the product. The Quarterly Journal of Experimental Psychology 56 A (1). 31-61.
García-Orza, J., Damas-López, J., Matas, A. y Rodríguez, J.M. (2009). " 2 x 3" primes naming " 6 ": Evidence from masked priming. Attention, Perception \& Psychophysics, 71 (3), 471-480.

Hecht, S. A. (1999). Individual solution processes while solving addition and multiplication math facts in adults. Memory \& Cognition, 27, 1097-1107.

LeFevre, J.A., Bisanz, J., Daley, K.E., Buffone, L., Greenham, S.L., y Sadesky, G.S. (1996). Multiple routes to solution of single-digit multiplication problems. Journal of Experimental Psychology: General, 125, 284-306.

LeFevre, J.A., Bisanz, J. y Mrkonjic, L. (1988). Cognitive arithmetic: Evidence for obligatory activation of arithmetic facts. Memory \& Cognition, 16, 45-53.

Lemaire, P., Barret, S.E., Fayol, M. y Abdí, H. (1994). Automatic activation of addition and multiplication facts in elementary school children. Journal of Experimental Child Psychology, 57, 224-258.

McCloskey, M. (1992). Cognitive mechanisms in numerical processing: Evidence from acquired dyscalculia. Cognition, 44, 107-157.

Romero, S.G., Rickard, T.C. y Bourne, L.E. (2006). Verification of multiplication facts: An investigation using retrospective protocols. American Journal of Psychology, 119, 87-121.

Rusconi, E., Galfano, G., Rebonato, E. y Umiltà (2006). Bidirectional links in the network of multiplication facts. Psychological Research, 70. 32-42.

Thibodeau, M.H., LeFevre, J., y Bisanz, J. (1996). The extension of the interference effect to multiplication. Canadian Journal of Experimental Psychology, 50, 393-396.

Winkelman, J.H. y Schmidt, J. (1974). Associative confusions in mental arithmetic. Journal of Experimental Psychology, 102, 734-736.

Zbrodoff, N.J., y Logan, G.D. (1986). On the autonomy of mental processes: A case study of arithmetic. Journal of Experimental Psychology: General, 115, 118-131.

Fecha de recepción: 23 de junio de 2009

Fecha de aceptación: 30 de julio de 2009 